\title{
Content, characteristics and function of mental images
}

\author{
Jean F. Fournier*, Sophie Deremaux, Marjorie Bernier \\ Institut National du Sport et de l'Education Physique, Laboratoire de Psychologie du Sport, 11, Avenue \\ du Tremblay, 75012 Paris, France \\ * Corresponding author. Tel.: +331417443 08; fax: +33141744535. \\ Article publié dans : Psychology of Sport and Exercise, 2008, vol. 9, $n^{\circ}$ 6, pp. 734-748 (doi : 10.1016/.psvchsport.2007.12.003)
}

\begin{abstract}
Objectives: The goal of this article is to better understand the use of mental imagery by athletes.

Design: Exploratory qualitative study followed by interviews with multimedia assessment tool.

Method: A first qualitative study was completed with two expert skydivers. The model obtained by the first study was tested in a second quantitative study with 32 skydivers. A multimedia tool was programmed to test the model so that films representing various content (internal or external views), characteristics (e.g., speed) and functions (e.g., to learn, to manage stress) was displayed during interviews in the second study.

Results: Results of the first study suggested that the use of imagery can be functionally explained by three related elements: content (what is visualized), characteristics (how it is visualized) and functions (why it is visualized) of mental images. Results of the second study showed that the content and the characteristics of imagery are linked to the function sought. Moreover, we found that the mental images used (content, characteristics and functions) varied according to the level of expertise (novice, confirmed or experts) and with the situation (when preparing for the jump, before jumping out of the plane or after the jump).

Conclusions: These results supplement the current stream of study, which has emphasized structural description of mental imagery since 1985. Our findings suggest that future research should build on structural dimensions of mental imagery and examine functional issues, because functional aspects have proved useful for understanding imagery use and for counseling athletes.
\end{abstract}

Keywords: Mental imagery; Function; Content; Characteristics; Sky diving

\section{Introduction}

Mental imagery is one of the most popular themes among both athletes and sport psychology researchers, probably because of its covert and thus mysterious aspects, combined with its overt superiority to no physical practice (Feltz \& Landers, 1983; Greenspan \& Feltz, 1989). This simple definition of imagery has been proposed by Hardy and Jones (1994, p. 67): "Imagery can be defined as a symbolic sensory experience that may occur in any sensory mode." Mental imagery has been considered similar to mental practice (Murphy \& Martin, 2002) and is a classic component of psychological skills training programs for athletes of various sports and levels (e.g., Bull, Albinson, \& Shambrook, 1996; Durand-Bush, Salmela, \& Green-Demers, 2001; Orlick, 1990). Cumming, Clark, Ste-Marie, McCullagh, and Hall (2005) returned to Marteniuk's (1976) definition of mental practice: 
"improvement in performance that results from an individual either thinking about a skill or watching someone else perform it".

Because of its wide practical applications and appeal, further research on imagery is clearly germane for athletes, sport psychologists, consultants, and coaches (Hardy, Jones, \& Gould, 1996).

Today, three major lines of imagery research are grounded in: (1) functional analysis of the brain (e.g., Decety \& Jeannerod, 1996), (2) the exploration of the functions of mental images (e.g., Martin, Moritz, \& Hall, 1999), and (3) comparison of real and imagined movement. The latter perspective is related to a motor learning paradigm. Authors study the ease or difficulty of preserving the temporal organization of the movement during motor imagery (e.g., Calmels, Holmes, Lopez, \& Naman, 2006; Guillot \& Collet, 2005). In this line of research, the goal is to study imagery duration, not functions. For example, instructions given to participants in Reeds (2002) were the following: "The divers were told to imagine themselves diving as if they were actually executing the dive or to mentally simulate their physical dive as closely as possible." Here, Reeds' participants are instructed to simulate diving as closely as possible in a training situation. The fact that during training sessions imagery duration lengthens with task complexity (Reeds, 2002) is not related to the purpose of our study. Hence, the present article is not part of the third line of research in mental imagery, nor the first, which is more closely related to neuroscience. The current article is part of the line of research exploring the functions of mental images.

Paivio (1985) published his classic article presenting a model of imagery functions, contending that imagery serves cognitive and motivational functions operating at general and specific levels. Paivio's model specifies that mental imagery influences behavior through four functions: (1) cognitive specific (CS), (2) cognitive general (CG), (3) motivational specific (MS), and (4) motivational general (MG). Examples of functions provided by Paivio are skill rehearsal (CS), strategy rehearsal (CG), goaloriented responses (MS), and self-confidence and arousal (MG).

This original model has since been both modified (Hall, Mack, Paivio, \& Hausenblas, 1998) and expanded (Martin et al., 1999) by the same team of researchers, but the core elements have remained. Hall et al. (1998) modified Paivio's model by specifying that the MG dimension was either oriented toward Mastery (MG-M) or toward Arousal (MG-A). The MG-M function relates to imagining performance in a confident manner to regain sport confidence, while the MG-A function serves to manage arousal and anxiety levels. According to Hall (2001), the current model of imagery functions (Hall et al., 1998) makes a persuasive case for matching each imagery function to an outcome. For example, if athletes want to increase their self-efficacy for performing a difficult skill, they should imagine themselves performing in a confident manner (MG-M) (Hall, 2001, p. 544). The Martin et al. (1999) model is the most recent extension. It supplements the model of imagery functions (Hall et al., 1998) and is composed of four hypotheses. The first is that athletes' mental imagery sessions take place in three different situations: training, competition and rehabilitation. Second, the model presents imagery types (CS, CG, MG-M, MG-A and MS), also called the function or the purpose that imagery is serving. The third hypothesis postulates three outcomes that athletes hope to achieve: (1) to learn skills and strategies for performance, (2) to modify cognitions and (3) to regulate arousal and competitive anxiety. Finally, Hall has recently stipulated that imagery is both a skill and an ability because imagery training leads to increases in the vividness, controllability and exactness of the mental images.

We were intrigued and concerned by the expansion of the original model of Paivio (1985). The original model has been modified over 20 years and has also applied to other sport psychology themes 
such as self-talk (Hardy, Gammage, \& Hall, 2001) or observational learning (Cumming et al., 2005). However, concepts and elements of the Martin et al.'s (1999) model overlap, a problem compounded by fluctuating terms and a general lack of operational definitions. Authors working with this model and the Sport Imagery Questionnaire (SIQ; Hall et al., 1998) tied to the model are starting to question such a model.

We consider it timely to highlight and illustrate some of the model's major drawbacks: Some concepts are jumbled as a result of a lack of operational definitions of the main elements (function, content and characteristics). We will now detail these problems in order to suggest some clarifications.

First, function has not been precisely defined. Authors use the words outcome and function interchangeably, so that when the model is applied to observational learning (Cumming et al., 2005), the findings do not follow Paivio's (1985) classification. Instead, Cumming et al. (2005) have found three functions: (1) motor skill acquisition and performance, (2) strategy development and execution, and (3) optimal arousal levels and mental states for performance. These functions are identical to the outcomes in Martin et al.'s (1999) model.

Moreover, function has also been associated with imagery type. In Martin et al.'s (1999) model, the central box was labeled "Imagery Type", while it was changed to "Imagery Function" by Hall (2001). However, in more recent studies (Callow \& Hardy, 2001; Short et al., 2002; Short, Monsma, \& Short, 2004), authors pointed out the need to clearly distinguish the type from the function. For example, Short et al. (2004) cited: "Callow and Hardy (2001) suggested that a differentiation must be made between the type of imagery (i.e., image content) a performer uses and the function it may have" (p. $342)$.

Second, imagery content has not been defined. Martin et al.'s (1999) "Applied Model of Imagery Use," makes no clear mention of the content, which is the answer to the question "What is visualized?" While the "What" question is central, these researchers makes no mention of content, since the model only deals with function. Indeed, the function of an image (e.g., enhancing confidence, MG-M) is assimilated to the image per se, while the image of a specific skill (CS) is appraised without its function (which could be to enhance learning but also to enhance confidence). Investigating whether an image of success (seeing oneself win or on the podium, MS) enhances confidence (an "outcome" in the model) lies at a different conceptual level than asking whether an image of feeling of confidence (MG-M) enhances confidence (outcome). In addition, authors have been overly tautological by writing that "to gain confidence, you need an image of gaining confidence (MG-M)". Many examples are available to illustrate this agreement among researchers, as seen in Martin et al. (1999, p. 250) or in Hall (2001, p. 544).

Recently, researchers have acknowledged that different participants may use the same image for different functions (Short et al. (2002) and Short, Monsma, \& Short (2004)). Rushall and Lippman (1998) made a related comment when they stated that athletes could adapt established skills to specific circumstances. It is likely that these authors meant functions by the word "circumstances" since they involved "memorizing" or "rehearsing" moves. A similar point was made by Calmels and Fournier (2001), who hypothesized that various imagery paces could serve different functions (e.g., learning skills, managing activation level in competition) in gymnastics.

Since 2001, the confusion between concepts in the original model has been clarified by some authors (Callow \& Hardy, 2001; Calmels \& Fournier, 2001; Nordin \& Cumming, 2005; Short et al. (2002) and Short, Monsma, \& Short (2004)). However, while the distinction between function, type and content has been confirmed by these authors, the confusion persists in the studies that still use the SIQ. The 
need for a revision of the SIQ has been expressed by this line of research. Short et al. (2004, p. 347) recently admitted that (citing Abma, Fry, Yuhua, \& Releya, 2002) "a limitation of the SIQ is that it does not tap into the athlete's perception of the purpose and goal of each type of imagery, and it assumes and requires that imagery content can be neatly classified into one of five categories (p. 73). Unfortunately, by instructing the participants in this study to select one function for each image, we made this very mistake!".

Third, the characteristics of imagery have never been defined. According to Hall (2001), content is composed of imagery quality such as vividness, when vividness is, so to speak, clearly a characteristic of a picture. However, the characteristics, or "how we mentally see", has not been conceptually distinguished from the other elements. In Munroe, Giacobbi, Hall, and Weinberg's (2000) study, there were four W's (The four W's of imagery use: where, when, why and what), but the how has never been addressed. Moreover, Munroe et al. stated that the content of imagery is to be categorized under various headings including once again imagery type. In this case, they present imagery type as visual or kinesthetic. However, kinesthetic or visual imagery are the two components of imagery ability in both Hall's (2001) and Martin et al.'s (1999) models, which suggest that imagery ability may moderate the link between imagery use and outcome. More recently, for Gregg, Nederhof, and Hall (2005), imagery ability refers to the quality of an individual's imagery. Not only do they specify that ability refers to visual or kinesthetic imagery, but they also rely on Hall (1998) and Hall and Martin (1997) in order to emphasize that the better imager an athlete is, the more effective his imagery. Here, authors are confounding two kinds of imagery (visual or kinesthetic) that provide different content of mental images (What) with the quality of a mental picture that relates to the characteristics (How) of the picture.

Considering all this original confusion and lack of definition of central themes (function, content, characteristics), we find it necessary to specify and distinguish the individual, overlapping concepts in play by posing the principle questions "why" and "what." Furthermore, the previous model did not explicitly distinguish the question of "how" the images are visualized. To differentiate among these three elements, the following definitions are suggested.

The function is why the mental images are imagined. For example, one may imagine in order to learn or to get motivated. The content of a mental image is what is imagined. It can be done with various senses: visual, (internal or external), auditory, kinesthetic, or olfactory. It may relate to various elements (e.g., the move, the outcome, the environment). Finally, the characteristics are how the mental image is imagined. Characteristics may involve various speeds, vividness or persistence.

The present research deals mostly with what is imagined by means of visual mental images, or visualization. To illustrate our aforementioned definitions, we use the TV analogy.

Why: Functions of mental images are defined as reasons for engaging in the visualization process. Functions are linked to the role attributed to mental images, or to the goal sought through the use of mental images. To fit the movie analogy, movies can be used to relax, to get aroused, to send a political message or to sell goods. In addition, real movies can be seen in various situations (i.e., during family time, at school, in a hardware store, on a plane, and also in a movie theatre).

What: The visual imagery of a sequence consists of "mentally" seeing a movie of a sequence, as could be produced on a TV screen. Therefore, the content depends on what has been filmed, whether it was a real subject or created with special effects. The content of a mental image is defined as what is mentally seen. Hence, films taken from an insider view (camera on a helmet) or from an outside view would provide content related to internal or external imagery perspective. 
How: To continue with the analogy of audiovisual equipment, the characteristics, or how the mental movie is seen, can be modified by the control commands on the TV and the DVD player. For example, vividness can be modified with the contrast and luminosity commands for the TV screen. Speed of the images can vary by accelerating or slowing down the movie to a complete pause with the control panel of the DVD player. In other words, characteristics of mental images relate to the elements that allow for distinguishing mental image quality.

The goal of this research is to test the validity of these definitions, in order to clarify the understanding of the concepts involved in mental imagery. Moreover, until now, the research about the "use" of imagery has been restricted to research on imagery functions (Gregg \& Hall, 2006). So the purpose of this study is to explore the functional relationships between the concepts (content, characteristics, functions) that allow for the understanding of the use of imagery by athletes. The previous structural classification of imagery use is considered a basis for this research. Gymnastics or figure skating are typically chosen for studying mental imagery, while other sports, such as sky diving, also emphasize morphokinetic skills. In skydiving, real practice can only be undertaken during the short duration of the fall. In addition, the 3D nature of the sport makes it impossible for athletes to simulate fully on the ground. Therefore, training and competing in this sport includes not only intensive use of video, but also requires intensive imagery practice to increase training time and to simulate the $3 \mathrm{D}$ performance elements. Exploring the use of mental images in elite sky diving was thus deemed pertinent because these athletes are most likely to be experts in imagery.

\section{Experiment 1}

The goal of the first study (qualitative) was to describe and analyze the use of mental imagery in two elite parachutists. We deliberately chose to involve such participants in order to observe how they use imagery compared to the Martin et al. (1999) original and modified models (Hall, 2001). Of course, the use of only two participants, even experts, does not provide much external validity to the findings. However, from a methodological perspective, there is only the need for one piece of evidence to demonstrate the existence of a phenomenon.

\section{Method}

\section{Participants}

Two of the world's best elite sky divers volunteered to participate in the study. To guarantee anonymity, the athletes are referred to as George and Mark. George is 33 years old and has been practicing this sport for 13 years, totaling over 6000 jumps, or $100 \mathrm{~h}$ of practice and competition. George has won the world championship, and is one of his country's highest ranked athletes. George's parachute specialty is relative work, a collective process with four teammates usually jumping at 4000 $\mathrm{m}$. They join arms and legs to execute a set of five different formations (figures in the shape of a donut, a caterpillar or a diamond, for example) as many times as possible before opening their parachutes, no lower than at $850 \mathrm{~m}$. The fall lasts about $60 \mathrm{~s}$.

The name and order of the set of formations are drawn and given to the athletes $1 \mathrm{~h}$ before the jump. George typically takes 15 min to pack his parachute, five to rehearse in a standing position the set they have been given, five for mental imagery, 10 to train lying flat on skateboards, and 10 more for mental practice. Depending on the order of the boarding call, George may use a $10 \mathrm{~min}$ pause to do more mental training before getting on the plane for a 15 min flight, during which he completes two additional minutes of imagery before jumping. George thus practiced mental imagery for about $17 \mathrm{~min}$ for each of his 6000 jumps, each lasting about $1 \mathrm{~min}$. It can be estimated that the $100 \mathrm{~h}$ of real practice 
have been supplemented by $1700 \mathrm{~h}$ of mental imagery practice. This figure does not consider imagery practice that took place between training sessions and competition.

Mark is 28 years old and has been practicing his sport for 10 years, totaling over 5000 jumps, or about $210 \mathrm{~h}$ of practice and competition. Mark is a world champion in canopy work and is his country's leader in this discipline. Teams of four athletes practicing canopy work usually jump at $2000 \mathrm{~m}$ and open their parachutes as they exit the plane. They execute various formations during the ride under their canopies by joining together and wrapping their feet in the ropes of the parachute underneath. The team must complete the set of formations as many times as possible before landing. The canopy ride lasts about $150 \mathrm{~s}$.

Competition conditions are identical to those of George. Mark typically takes 15 min to pack the parachute and 10 to rehearse the drawn set in a standing position, while practicing mentally, followed by another $10 \mathrm{~min}$ of practicing mentally alone. While waiting for takeoff, Mark executes several 1 min periods of mental imagery. Five minutes before getting on the plane, the whole team rehearses while standing up one last time. Once in the plane, Mark spends over 3 min doing mental imagery until the plane reaches jump altitude. In short, Mark practiced mental imagery for about 23 min for each of his 5000 jumps, each lasting $2.5 \mathrm{~min}$. It can therefore be estimated that his $210 \mathrm{~h}$ of practice have been supplemented by $1900 \mathrm{~h}$ of mental imagery. For both George and Mark imagery was selftaught and used spontaneously, without external help, which is generally the case for skydivers, who rely upon this skill from the very beginning of their careers.

\section{Interview guide}

Semi-structured, qualitative interviews were used to collect data on the mental imagery experiences of the participants during training and competition for both athletes. Questions and probes were based upon "The four Ws of imagery use: Where, When, Why, and What" (Munroe et al., 2000). The following themes were addressed during the interviews: (a) biographical information (e.g., name, surname, age, number of years of practice, sport background and results), (b) description of real practice (e.g., training load, number of jumps, description of the steps undertaken before jumping, description of the jump itself), (c) description of the role/importance of mental practice (e.g., efficacy of imagery use in his practice of skydiving, imagery training), (d) description of the mental images used and of their characteristics (e.g., what is visualized, from what perspective, what speed, what quality and control of the pictures, what imagined sensations), (e) description of time and frequency of imagery use (e.g., when, how long, at what frequency), and (f) exploration of the reasons invoked for each use of imagery. The full interview guide is available upon request from the authors.

\section{Procedure}

The interviewer executed a pilot study on one male and one female skydiver competing at the national level. The semi-structured interviews each lasted $1 \mathrm{~h}$. Following the pilot study, we decided to make sure all the topics were addressed, but in the order chosen by the participants so as to follow the flow of their discourse. Permission was requested and granted to record the interviews.

Interviews began by recalling the purpose of the study, while a definition of mental imagery was given to specify and emphasize the theme of the interview. The interviewer made sure that all interview themes were addressed for each participant. Each interview lasted 90 min and resulted in 16 pages of verbatim transcription for George's and 17 pages for Mark's. Upon assessing the two transcripts, we noted that the description of the characteristics of George's mental images were not exhaustive. 
Therefore, 3 weeks later, an additional 30-min interview was conducted with George to supplement data regarding mental imagery use during training.

\section{Validity, trustworthiness}

The results of the present research can be considered trustworthy because they meet most of Sparkes's (1998) criteria in the design, data collection and analysis. Credibility was addressed (member checks, prolonged engagement, triangulation), transferability (thick description) and confirmability. Interview transcripts were returned to athletes for them to supplement or modify their reports. Neither considered that the transcript needed changing. By doing this, the credibility criteria were fulfilled through member checking.

The researcher who conducted the interviews holds a Masters degree in sport psychology and was trained in interviewing skills. The interviewer was a member of the national team, which has been ranked in the top three in the world. The fact that she was participating in the sport at the elite level along with the participants allowed for establishing credibility through prolonged engagement and persistent observation.

Three analysts independently completed the inductive coding of the transcripts and this collective experience fostered an emotional detachment from both the sport and the participants. The first researcher had been using qualitative analysis for over 10 years, while the second had undergone three years of training qualitative research. The third sport psychology researcher (interested in attributions and from a different institution) performed an independent audit. This analyst understood elite athlete populations (e.g., triathletes), was unaware of mental imagery and skydiving, but received coding instructions of the transcripts.

\section{Data analysis}

Analysis was processed in three stages, beginning by processing the inductive coding of the meaning units (MUs), while keeping in mind Munroe et al.'s (2000) four questions regarding imagery use: where, when, why, and what. These questions were not only helpful in designing the interview guide, but also in processing the analysis.

In the first stage, we processed an inductive coding of the MUs. This stage allowed us to select MUs regarding what participants said they were doing. Inter-observer agreement on the codes (Thomas \& Nelson, 1996) reached $96 \%$. When participants described "what" they were imaging, it was found that both took time to describe the characteristics of mental images, or "how" they were imaging. Special attention was paid to the characteristics of each mental image reported by the participants, as represented by the tags for each MU (Côté, Salmela, \& Baria, 1993).

In the second stage, tags with similar meaning were gathered within the same category and were examined in relation to the functions attributed to each MU. This step was useful to gain understanding about "why" the participants used mental images with varied content. Properties of the categories were examined in light of answers to Munroe et al.'s (2000) four questions, as well as the question "how."

During the third stage, we tried to appraise relationships among the content, the characteristics of mental pictures, and the functions. We explored and described what images were used and for what reason. 


\section{Results}

The first stage of the analysis permitted the selection of 840 MUs related to mental imagery. In the second stage, the MUs were regrouped into 44 tags, which subsequently resulted in three categories. These three categories of mental imagery were labeled as content (what is imaged), characteristics (how is it imaged) and function (why is it imaged). All 840 MUs and 44 tags derived from the transcripts and were classified into one of the three categories.

In the third stage, a dynamic relationship among the content (Co), the characteristics (Cha) and the functions (F) of mental images was found. Since the sample was only composed of two elite participants, we were only able to suggest that a relationship of the external validity should be studied with a larger sample. Often, excerpts that were remote from one another in the transcripts were clearly related to the same situations. We will now present the results of the analysis by illustrating these relationships with various situational examples for the two participants.

\section{Analysis of George's transcript}

Once the collective briefing was over, George started his first imagery session, on the ground. He had to find the best solution to the problem caused by the draw (function, F). To accomplish this goal, George visualized various options of the complete sequence, with an external perspective (content, Co) of slow and blurry pictures (characteristics, Cha).

When I visualize from the ground, I have to learn the order of the formation in the sequence. It's rather global because I'm not precise in the moves connecting one formation to the other. I see the fall from above, that is, in fact, I see myself move. What's good about this is that it allows me to visualize the whole sequence, so I can see the entire jump. In this case, when I'm trying to understand a sequence, my pictures are a bit dim, not very sharp. I fiddle a little, I play with my images, I modify them if needed, I correct them until they are perfect, accurate. In fact, in the beginning, images come rather slowly. That means, the routine we rehearsed mentally, that we visualized, is going to be a bit slower at the beginning. It's going to be slower than the real speed, to understand the sequence.

Once the sequence was "understood," i.e., once the best connection between each formation was selected from among different pictures (Co), the quality or vividness (Cha) of the mental pictures started to increase. As the function of the imagery changed to memorizing $(F)$, the speed of the mental movie increased to reach the real speed (Cha).

I visualize the whole sequence, the pictures are still slow, more precise, so that I can memorize more efficiently, to remember everything. Once the sequence is known by heart, I connect the formations with the real speed to get as close as possible to the actual jump.

However, in some cases, the outcome of the draw may have required the execution of sequences that were more complex than usual. The difficulty lay in the connection between the formations, and more specifically, in the individual moves in relation to his partners' moves. George had to focus on these moves so that they were easier to learn (F). He visualized mostly the grip (Co), by blowing-up the internal picture of his hands and by pausing the mental movie (Cha).

For some pictures of tricky moves, I especially see my arms and hands. In my visualization I will pause longer than in real time. In fact, I see more specifically this part of my body. I see it a bit larger, to pay better attention. 
To learn a difficult sequence, George modified the characteristics of his mental pictures to better focus on specific elements. Other modifications of mental pictures might also have involved a change in perspective, and hence the content of the picture: instead of seeing his own arms grabbing the grips, George saw the whole team performing $(\mathrm{Co})$.

When I visualize and I can't find the solution, I'll go for the upper view. In fact, I'll try to place myself in the videoman's position. This is another way to enhance my performance; it strengthens my self-confidence.

Changing from the internal to the external perspective seems here not only to serve the same function, but might also have boosted confidence (F). Other excerpts throw light on the dual function (learning and increasing confidence) served by different image content:

I first visualize my personal moves, to check if everything is accurate, and then I visualize the other teammates' moves. This gives me a perfect understanding of the jump. And also, it reassures me.

Originally, the function of the visualization was to learn a move, but confidence also increased. This example shows that the use of two pictures (seeing one athlete and seeing the whole team) may have simultaneously served two functions (learning and enhancing confidence). Going back to the chronology of the jump preparation, we understood that the content and the characteristics of the mental pictures varied according to the new functions sought. This was particularly noticeable as the participant got closer to the onset of the jump. During the plane ride to reach the flight level, functions were more closely related to both arousal and attention control. Only the beginning of the sequence was visualized (Co), at a fast-paced rate (Cha). The speed of the mental movie is perceived as being quicker than real speed, which contributes to increasing his arousal level.

Right before jumping, I rehearse the first two formations. I shut myself off to be ready right away. It's just before jumping, to get immersed in the move, like a start before the start, here, my pictures come quickly, they're fast. It kind of pumps me up, you know, to end up ready for the jump.

\section{Analysis of Mark's transcript}

Mark's practice also entailed a large imagery component. He was in the habit of visualizing before each jump in the following chronological steps. During the first briefing following the draw, Mark simultaneously visualized all the individual moves that connect formations (Co). This visualization was done while mimicking the whole sequence in a standing position, with his arms raised to simulate steering the canopy. This first session was meant to vertically transpose the moves executed horizontally by the team on the floor, i.e., to understand the sequence (F). Only through visualization could Mark portray the moves in three dimensions. To reach this goal, Mark used an external mental movie, in black and white (Cha).

While we are doing the moves on the floor, I've got to picture the trajectories vertically, as they will be during the ride. I have to transpose vertically the floor trajectories and positions that we have done horizontally, so that I can imagine them in my head, on a vertical plane, in the air.

Once the briefing was over, Mark started his second set of imagery sessions. He was alone, generally in the packing area. He physically simulated the onset of each of his moves for each formation. However, the content and the characteristics of the mental movies were specific to the difficulty of the 
sequence. In a simple sequence, Mark stayed in the same position and used a few standardized connection moves between the formations. Visualization involved three steps executed in 10 min: (1) Mark described first seeing a succession of black and white still pictures (Cha) of the five formations (Co) as being projected on a 20 in screen to learn the order of the formations $(F),(2)$ then, mental movies (not paused images) (Cha) of all his teammates' moves $(\mathrm{Co})$ were visualized to understand the connection between the formations (F), (3) and third, the focus of the mental movies (Cha) switched to personal moves, positions, marks, trajectories and grip (Co), allowing him not only to understand his own moves $(\mathrm{F})$ but also to gain confidence $(\mathrm{F})$.

In a complex sequence, Mark had three strategies. First, he could use indirect trajectories so that his less skilled teammates could execute the simpler ones. Or he could execute the sequence in a position other than his own, or in a different one for each formation. In each of these cases, visualization involved the same first steps, and an additional second step, executed in $15 \mathrm{~min}$. Characteristics of the movies were modified for the second step: the athletes, actors of the mental movie, were deliberately blurry, but the canopies were clear; (Cha) each individual move was visualized in its entirety (Co) so that Mark could understand all of the connection moves (F). This was rather efficient when Mark had to change positions with every new formation: the two-step visualization strategy allowed him to learn the whole sequence with all of the individual moves once and for all, instead of learning each position leading to all teammates for each formation.

Once on the plane, during take-off, Mark visualized slowly (Cha), from an external perspective (Co) and in great detail, all the teammates' moves and positions (Co), along with the specificities related to his own position (Co) from an internal perspective (Co). The scope of the visualization was limited to the sight of his partners (Co), thus excluding opponents who could be present on the plane. Mark contended that this helped him control distractions $(\mathrm{F})$, while staying calm $(\mathrm{F})$ and confident $(\mathrm{F})$. As the jump got closer, the mental picture took on colors (Cha). To strengthen distraction control, Mark closed his eyes:

I review everything mentally, the trajectories, what I must do, where I must go. I see all my teammates by the plane door - the opening - where the beginning of the formation should be, where my teammates should be, the trajectories they'll follow when exiting the plane. I see the four of us, others don't exist anymore. My mental images are faster than when I'm learning, because I already know the jump. That prevents me from thinking about useless stuff, such as, um-the score or a failure. It's more to keep myself alone, to brush up on my trajectories.

At $1500 \mathrm{~m}, 5 \mathrm{~min}$ before the jump, Mark wanted to remain calm and to avoid worrying about forgetting a move between two formations. He also wanted to remember all the elements of the sequence and thus needed to review the sequence (F). He visualized the complete sequence (Co), but this time with high speed, images in color and from an internal perspective (Co).

At the end, just before jumping, images come to my head super fast, crystal-clear and my eyes are wide open, yet my pictures are clear. I see the complete sequence, but really from inside. This allows me to stay alone, to shut myself off, to keep the pressure from building up. I don't want to get stuck in a memory lapse, but because I do know the sequence, I don't worry about that.

It is clear in this case that the same image content was used with a given set of characteristics to achieve more than one function. 
In summary, distinctions within the content, characteristics, and functions of mental images were found. Moreover, this research indicates that elite athletes who participated in this research used imagery for different functions that dictated the content and the characteristics of their mental images.

\section{Discussion}

The goal of this study was to describe and understand the use of mental imagery. Two elite parachutists volunteered to be interviewed regarding their mental practice in general, and more specifically, about visualization. It appeared that the questions where and when led to the same answers, and were related to the situation. This is congruent with Munroe et al.'s (2000) findings, since both training and competition situations are gathered under the when and where components of their analysis. This first exploratory study enabled us to classify all imagery use with the three elements that were defined (Content, Characteristics and Functions).

Different situations highlighted by the participants (i.e., during training, during competition, during difficult or easy moves) showed that the three elements were dynamically related. However, "function" seemed to be the key element responsible for the setting of the content and the characteristics of the mental images. For example, when the goal was to learn a new sequence (once the draw was known), the mental image content might be some blurry, schematic shapes representing the parachutists performing at a slower pace. When the function changed to increasing the activation level (right before the jump) and maintaining confidence, the content and the characteristics varied to match the requirements. Mental movies were then imaged from an internal perspective, at a pace faster than the real speed. Therefore, for these two athletes, the function sought determined the content and the characteristics of the mental images, presumably to reach the desired outcome. In summary, it was observed that the content and the characteristics of mental images were generated because they corresponded to specific needs, or functions, in specific situations (i.e., during training or competition, easy or difficult sequences, or different steps of the preparation of the fall).

\section{Experiment 2}

In the first study, we observed that some content and characteristics of mental images corresponded to specific functions. Since there were only two participants (elite athletes), we extended this description in a second study, without yet generating hypotheses. The study was performed on a larger sample of parachutists of various levels to seek external validity through the means of a multimedia tool. To get a better understanding of imagery use and of the relationships among content, characteristics and functions of mental images, we designed a computer program called Skydive. This multimedia tool was deemed helpful to get a deeper understanding of mental imagery use over a large sample of participants. Skydive was therefore programmed so that the three elements of mental images could be appraised with regard to objective sport moves in motion.

\section{Method}

\section{Participants}

Thirty-two skydivers volunteered to participate in the study. Codes were used to protect anonymity of computer data, with respect to legal and ethical considerations. Participants were gathered into three groups based on their level of practice. Ten experts (mean age 31) belonged to national teams and had participated in international tournaments. Eight held international titles. They averaged 5615 jumps 
and 11.7 years of practice. Ten experienced participants (mean age 30) had competed in national tournaments for a minimum of 3 years. They averaged 1485 jumps and 9.7 years of practice. Twelve beginners (mean age 27) averaged 266 jumps and 2.7 years of practice.

\section{Tool}

Skydive was programmed in HTML format to present the questions and display the answers. This multimedia tool functions with a Java applet that saves scores of the HTML form in a file on the local hard disk and a Quick Time plug-in for playing movies. An Internet browser is required to use Skydive. Participants are asked to visualize four different sky diving situations, then to select the content, characteristics and functions of the mental images they have just used from among different options. All answer choices of the multimedia tool used elements from interviews with the experts of the first study. For example, one skydiver of the first study stated that he used black and white, so we included this movie option. For this second study, an "other" option was made available, so that participants could add other characteristics. The "other" option was selected four times during interviews, and these additions were later classified under pre-existing choices.

To control inter-individual variability of answers (to present a norm), answer options are facilitated by movies (various content and characteristics) of a team performing the fall. Participants were able to choose their answers between internal or external movies. Internal movies were made from a camera placed on the helmet of a member of the team performing the fall. External movies were obtained from the cameraman filming the team from above. For each perspective, participants were able to play four movies of different speeds (slower than normal, normal, faster, and with speed variations), three movies of different quality (very blurry, a bit blurry and normal) and between black and white or color. Movies were altered in size, speed, color and vividness (contrast and luminosity) with Adobe Premiere software. The clips obtained were saved in QuickTime format to be used in the HTML form. All movies were displayed simultaneously on the same screen inside square windows. Window size was 130 pixels, which corresponds to $4 \mathrm{~cm}$ squares on a 15 in screen monitor set to display $800 \times 600$ pixels. Participants could select the function they attributed to their mental images from the following choices: to learn, to manage stress, to get energized, to be motivated, to focus, to correct moves, for confidence. The tool was first pilot tested on 10 parachutists, on a 15-in screen laptop computer. One movie was discovered to be misplaced. Once the correction was made, the tool was considered satisfactory for the purpose of the study. Skydive can be obtained through the first author.

\section{Procedure}

Individual interviews with Skydive lasted $22.5 \mathrm{~min}$ on average. The experimenter was present to help the participant use the software and to answer questions. The following procedure was used: (a) the introduction of the experimenter, (b) the presentation of the goal of the study (explore participant imagery use) and of the multimedia tool, (c) the giving of an example of mental imagery, and (d) a reminder of the anonymity of the answers, of the possibility to ask questions at anytime and to modify answers if not satisfied. Once in front of the computer, participants had to enter biographical data. They were then asked to visualize four situations related to the preparation of the jump preparation: (1) discovering the draw of the jump, (2) after preparing the jump on the skate board, (3) in the plane, 5 min before the jump, and (4) after the jump, once on the ground. After each situation was visualized, participants were asked to select movies that best corresponded to the content and characteristics of the images they had just visualized. Participants also had to select a function corresponding to the reason they engaged in the visualization process in the given situation. 


\section{Results}

Data collection consisted in 147 observations (mental pictures) associated with their characteristics and one or two functions each. These observations allow for the explanation of mental imagery use found in study 1. A $\chi 2$ test was processed to examine links among various qualitative variables: sport level, content (and perspective), speed, vividness, color and functions. A significant global $\chi^{2}$ indicates a link between two qualitative variables. When the $\chi^{2}$ was significant, a $\chi 2$ per cell (Grimmer, 2004) was calculated and interpreted. $\chi^{2}$ per cell (one degree of freedom) is a sort of post hoc test for global $\chi^{2}$ that allows for detecting independence or links between two levels of two given qualitative variables. Each of the four cells of the contingency table was studied. This allowed us to test the link between two levels, in the four-cell contingency table. We computed 10 global $\chi 2$ 's and $149 \chi 2$ 's per cell (total of 159 analyses). Because in the present study we were interested mostly in functional links between concepts (and not in structural links, for example, between content and characteristics), we computed global $\chi 2$ 's to test the relationship between:

1 - situation and function, followed by $28 \chi 2$ 's per cell,

2- function and content, followed by $14 \chi^{2}$ 's per cell,

3 - speed and function, followed by $21 \chi 2^{\prime}$ s per cell,

4 - vividness and function, followed by $21 \chi 2$ 's per cell,

5 - color and function, followed by $14 \chi 2$ 's per cell,

6 - level of expertise and content, followed by six $\chi 2$ 's per cell,

7 - level of expertise and function, followed by $21 \chi 2$ 's per cell,

8 - level of expertise and speed, followed by nine $\chi 2$ 's per cell,

9 - level of expertise and vividness, followed by nine $\chi 2$ 's per cell,

10 - level of expertise and color, followed by six $\chi 2$ 's per cell.

Out of these 159 analyses, we choose to present the 10 global $\chi 2$ 's, and significant $\chi 2$ 's per cell.

\section{Links between situation, functions, content and characteristics}

\section{Links between situation and function of mental images}

When all four situations are taken together, there is a significant link between situation and function of mental pictures; $\chi^{2}(18,129)=162.28 ; \mathrm{p}<.00$. In the first situation (discovering the draw) the function "to learn" is over-represented; $\chi^{2}$ per cell $(18,129)=11.21 ; \mathrm{p}<.01$. For all observations in situation 1 , mental imagery is used to learn $73 \%$ of the time. In the second situation (after preparing the jump on the skateboard) the function "to learn" is over-represented, $\chi^{2}$ per cell $(18,129)=2,83 ; p<.05$. For all observations in situation 2, mental imagery is used to learn $52 \%$ of the time. In the third situation (in the plane, $5 \mathrm{~min}$ before the jump) the function "to concentrate" is over-represented, $\chi^{2}$ per cell $(18,129)=7.71 ; \mathrm{p}<.01$. For all observations in situation 3 , mental imagery is used $50 \%$ of the time to concentrate. The function "to manage stress" is over-represented, too, $\chi 2$ per cell $(18,129)=6.35$; $\mathrm{p}<.01$. Mental imagery is used $17.50 \%$ of the time to manage stress. In the fourth situation (after the jump, once on the ground) the function "to correct the jump" is over-represented; $\chi^{2}$ per cell 
$(18,129)=71.84 ; \mathrm{p}<.01$. For all observations in situation 4 , mental imagery is used to correct the jump $95 \%$ of the time.

\section{Links between function and content (perspective) of mental images}

When all four situations are taken together, there is a significant link between function and content (perspective) of mental pictures; $\chi 2(7,129)=13.29 ; \mathrm{p}=.04$. When the function is "to correct the jump", internal perspective is over-represented; $\chi^{2}$ per cell $(7,129)=2.77 ; \mathrm{p}<.05$. When mental imagery is used to correct the jump, perspective of images is internal $76 \%$ of the time. When the function is "to correct the jump", external perspective is under-represented; $\chi 2$ per cell $(7,129)=2.82 ; p<.05$. When mental imagery is used to correct the jump, perspective of images is external $24 \%$ of the time.

\section{Links between characteristics and functions of mental images}

Three characteristics of mental imagery and their links with function were studied: speed, vividness and colors.

Links between speed and function: When all four situations are considered together, there is a significant link between speed and function of mental pictures; $\chi 2(18,129)=39.79 ; p<.01$. When the function is to learn, fast speed is under-represented; $\chi^{2}$ per cell $(18,129)=4.71 ; p<.01$. When mental imagery is used to learn, the speed is fast only $2 \%$ of the time. When the function is "to manage stress", the fast speed is over-represented; $\chi^{2}$ per cell $(18,129)=11.16 ; p<.01$. When mental imagery is used to manage stress, the speed is fast $28 \%$ of the time.

Link between vividness and function: When all four situations are taken together, there is no significant link between vividness and function of mental pictures; $\chi 2(12,127)=10.82 ; \mathrm{p}=.54$.

Link between colors and function: When all four situations are taken together, there is no significant link between color and function of mental pictures; $\chi 2(6,129)=6.43 ; p=.38$.

\section{Differences in use of mental imagery according to level of skydiving expertise}

\section{Links between content of images (perspective) and level of expertise of the skydivers}

When all four situations and all three levels of expertise are considered together, there is a significant link between content of images and level of expertise; $\chi 2(4,147)=31.84 ; p<.01$. For the beginners, external perspective is over-represented; $\chi 2$ per cell $(4,147)=3.52 ; \mathrm{p}<.01$. For all observations of the beginners, external perspective is used $61 \%$ of the time. The lack of use of imagery is overrepresented; $\chi^{2}$ per cell $(4,147)=7.08 ; \mathrm{p}<.01$. The beginners did not use imagery in $24 \%$ of the observations. For the experts, internal perspective is over-represented; $\chi^{2}$ per cell $(4,147)=4.06 ; p<.01$. For all observations of the experts, internal perspective is used $64 \%$ of the time.

\section{Links between function of images and level of skydiving expertise}

When all four situations and all three levels of expertise are taken together, there is a significant link between function of images and level of expertise; $\chi^{2}(12,129)=28.05 ; \mathrm{p}<.05$. For the beginners, the function "to manage stress" is over-represented; $\chi^{2}$ per cell $(12,129)=7.56 ; p<.01$. For all observations of the beginners, imagery is used $19 \%$ of the time to manage stress. For the experts, the function "to activate" is over-represented; $\chi^{2}$ per cell $(12,129)=3.62 ; p<.05$. For all observations of the experts, imagery is used $11 \%$ of the time to activate. 
Links between speed of the images and level of expertise: When all four situations and all three levels of expertise are taken together, there is a significant link between speed of images and level of expertise, $\chi^{2}(6,130)=19.90 ; p<.01$. For the beginners, the slow speed is over-represented; $\chi^{2}$ per cell $(6,130)=5.84 ; p<.01$. For all observations of the beginners, the slow speed is used $43 \%$ of the time. The use of a variation of the speed (acceleration or slow motion) is under-represented; $\chi^{2}$ per cell $(6,130)=4.55 ; \mathrm{p}<.05$. The beginners did not vary at all the speed of the images.

Links between vividness of the images and level of expertise: When all four situations and all three levels of expertise are taken together, there is a significant link between vividness of images and level of expertise; $\chi^{2}(4,128)=46.47 ; \mathrm{p}<.00$. For the beginners, very blurred images are over-represented; $\chi^{2}$ per cell $(4,128)=18.37 ; \mathrm{p}<.01$. For all observations of the beginners, images are very blurred $28 \%$ of the time. For the experts, the normal vividness of images is over-represented; $\chi 2$ per cell $(4,128)=6.72$; $\mathrm{p}<.01$. For all observations of the experts, images are in a normal vividness $56 \%$ of the time.

Links between color of images and level of expertise: When all four situations and all three levels of expertise are considered together, there is a significant link between color of images and level of expertise; $\chi^{2}(2,130)=16.61 ; \mathrm{p}<.001$. For the beginners, images in black and white are overrepresented; $\chi^{2}$ per cell $(2,130)=9.00 ; p<.01$. For all observations of the beginners, images are in black and white $43 \%$ of the time. For the experts, images in colors are over-represented; $\chi^{2}$ per cell $(2,130)=0.94 ; p<.05$. For all observations of the experts, images are in color $92 \%$ of the time.

\section{Discussion}

Statistical analysis highlights various functional links among the three elements (content, characteristics and functions). Depending on the situation (e.g., in the plane, before jumping or on the ground after the jump) there is a link with the function of mental images. Skydivers visualize more to focus on the moves before the jump and more to correct their move after. These two functions are directly linked to what skydivers visualize (the content), since their goals differ in the two situations. For example, they use more of the internal perspective (content) to correct the next jump. Similarly, there is a link between the function of the mental images (why they are used) and their characteristics (how they are pictured). Regardless of the level of expertise, skydivers visualize slowly to learn a move and quickly to manage their stress. This variation of mental pictures in relation to function has already been mentioned by Rushall and Lippman (1998). Hence, it appears that the three elements, while interrelated, vary with the demands of the situation. A second main finding is that the level of expertise is linked to specific imagery use. Novice, confirmed or expert skydivers do not use mental imagery in the same way. Since functions change with experience, the content and characteristics vary accordingly. Beginners need to learn their moves differently than experts, hence the content of their mental imagery is different. Of course, the different content and characteristics of mental pictures may also be related to the imagery skill potentially acquired with experience both in skydiving and imagery practice for this specific sport. For example, using one perspective might be related to imagery ability (e.g., novices may not be capable of using internal perspective). The level of expertise in skydiving could have also an impact on which perspective is chosen to provide specific information to the athlete. In addition, the difference in the use of characteristics may come from his level of expertise in imagery and in skydiving, or from a deliberate choice on the athlete's part. For example, an athlete may avoid seeing clearly when emphasizing another characteristic of the mental image, such as speed. In this case, there might be a voluntary trade-off between vividness and speed (Guillot \& Collet, 2005). 


\section{Overall summary and conclusion}

In the introduction, we noted the confusion and the lack of definition of central themes (function, content, characteristics). We specified and distinguished the individual, overlapping concepts in play by posing the principle questions "what" and "why." We mentioned that the previous model did not explicitly distinguish the question of "how" the images are visualized.

It was hypothesized that three higher order themes could help classify codes related to content, characteristics and functions of mental images. In the first qualitative study, we found classification and the definitions pertinent to examine imagery use by two elite skydivers. The second study extended the results to 32 skydivers. With the help of a multimedia tool, the participants were able to describe their use of mental images in four situations. Different uses of imagery were observed in these four specific situations and various types of imagery use depend on the level of expertise (novices, confirmed, experts).

With these two studies, we observed that content and characteristics fit the function of the mental images. This became clear in various situations (training, competition, difficult moves and various stages of jump preparation) and for different levels of expertise in imagery and skydiving.

Hence, we have moved from the current conceptual framework for athletes' use of imagery (Munroe et al., 2000), summarized in Fig. 1, to a more dynamic association of elements that describes imagery use: "A situation leads to a function that initiates mental image content, with characteristics". We hereby proffer an alternative model of mental imagery use (Fig. 2), based on functional aspect rather than on a structural one. The core of the working model we offer emphasizes dynamic relationships (represented by arrows) between the elements, and adds the "characteristic" element. This novel distinction between "what" and "how" in our working model offers new research perspectives to enhance the knowledge about the structural dimension of mental imagery.

In our alternative working model, function is a central concept. According to Boy (1998), functions are not organized in terms of input or output, as is the case for process; functions are defined by the task. The structure of a function resembles that of a task, if one had to analyze the task. Functions are sensitive to context and they impact the representation required to accomplish the goal of the task. We therefore claim that imagery is primarily functional, and secondarily structural. We suggest that it might be time for research to build upon the description of the structure of mental imagery and to start examining the relationship between content, characteristics and the functions they serve. This new direction of research might be useful for sport psychology services because it would allow for targeting advice related to a given function for individuals dealing with a specific situation.

Results from this research need to be replicated and confirmed on a larger scale with other elite participants. If it is confirmed that function initiates the content and characteristics of mental images, future investigation should try to evaluate which kind of content and characteristics are more efficient for the various functions sought by athletes at different skill levels. Not only is this important for theoretical purposes, but also for advising athletes. This implies that research and application should move towards enhancing this more dynamic model of imagery function, taking into account relationships among content (what), characteristics (how) and function (why).

\section{Acknowledgements}

We would like to thank George Calder for his comments on study 1, Soizic Toutirais for the external audit, Laura Killian for her corrections, and Brice Lefevre and Paul Fontayne for their contribution to the statistics. 
Parts of this paper were presented at the 2003 conference of the Association for the Advancement of Applied Sport Psychology.

\section{References}

Abma, Fry, Yuhua, \& Releya (2002). Difference in imagery content and imagery hability between high and low confident track and field athletes. Journal of Applied Sport Psychology, 14 (2002), pp. 67-75

Boy (1998). Cognitive function analysis. Ablex Publishing, Wesport (1998)

Bull, Albinson, \& Shambrook (1996). The mental game plan: Getting psyched for sport. Sports Dynamics, Eastbourne (1996)

Callow \& Hardy (2001). Types of imagery associated with sport confidence in netball players of varying skill levels. Journal of Applied Sport Psychology, 13 (2001), pp. 1-17

Calmels \& Fournier (2001). Duration of physical and mental execution of gymnastic routines. The Sport Psychologist, 15 (2001), pp. 142-150

Calmels, Holmes, Lopez, \& Naman (2006). Chronometric comparison of actual and imaged complex movement patterns. Journal of Motor Behavior, 38 (2006), pp. 339-348

Côté, Salmela, \& Baria (1993). Organizing and interpreting unstructured qualitative data. The Sport Psychologist, 7 (1993), pp. 127-137

Cumming, Clark, Ste-Marie, McCullagh, \& Hall (2005). The functions of observational learning questionnaire (FOLQ). Psychology of Sport and Exercise, 6 (2005), pp. 517-537

Decety \& Jeannerod (1996). Mentally simulated movements in virtual reality: Does Fitts law hold in motor imagery?. Behavioral Brain Research, 72 (1996), pp. 127-134

Durand-Bush, Salmela, \& Green-Demers (2001). The Ottawa Mental Skills Assessment Tool (OMSAT-3). The Sport Psychologist, 15 (2001), pp. 1-15

Feltz \& Landers (1983). The effects of mental practice on motor skill learning and performance: A metaanalysis. Journal of Sport Psychology, 5 (1983), pp. 25-57

Greenspan \& Feltz (1989). Psychological interventions with athletes in competitive situations: A review. The Sport Psychologist, 3 (1989), pp. 219-236

Gregg \& Hall (2006). The relationship of skill level and age to the use of imagery by golfers. Journal of Applied Sport Psychology, 18 (2006), pp. 363-375

Gregg, Hall, \& Nederhof (2005). The imagery ability, imagery use and performance relationship. The Sport Psychologist, 19 (2005), pp. 93-99

Grimmer (2004). Statbox (Version 6.4). Grimmer Soft, Paris (2004) [Computer Software Paris]

Guillot \& Collet (2005). Duration of mentally simulated movement: A review. Journal of Motor Behavior, 37 (2005), pp. 10-20

Hall (1998). Measuring imagery ability and imagery use. J.L. Duda (Ed.), Advances in sport and exercise psychology measurement, Fitness Information Technology, Morgantown, WV (1998), pp. 165-172 
Hall (2001). Imagery in sport and exercise. R. Singer, H.A. Hausenblas, C.M. Janelle (Eds.), Handbook of sport psychology, Wiley, New York (2001), pp. 529-549

Hall, Mack, Paivio, \& Hausenblas (1998). Imagery use by athletes: Development of the Sport Imagery Questionnaire International Journal of Sport Psychology, 29 (1998), pp. 73-89

Hall \& Martin (1997). Measuring movement imagery abilities: A revision of movement imagery questionnaire. Journal of Mental Imagery, 21 (1997), pp. 143-154

Hardy, Gammage, \& Hall (2001). A description of athlete self-talk. The Sport Psychologist, 15 (2001), pp. 306318

Hardy \& Jones (1994). Current issues and future directions for performance-related research in sport psychology. Journal of Sport Sciences, 12 (1994), pp. 61-92

Hardy, Jones, \& Gould (1996). Understanding psychological preparation for sport. Theory and practice of elite performers. Wiley, Chichester (1996)

Marteniuk (1976). Information processing in motor skills. Holt, Rinehart and Winston, New York (1976)

Martin, Moritz, \& Hall (1999). Imagery use in sport: A literature review and applied model. The Sport Psychologist, 13 (1999), pp. 245-268

Munroe, Giacobbi, Hall, \& Weinberg (2000). The four Ws of imagery use: Where, when, why and what. The Sport Psychologist, 14 (2000), pp. 119-137

Murphy \& Martin (2002). The use of imagery in sport. T.S. Horn (Ed.), Advances in sport psychology, Human Kinetics, Champaign, IL (2002), pp. 405-439

Nordin \& Cumming (2005). Professional dancers describe their imagery: where, when, what, why and how. The Sport Psychologist, 19 (2005), pp. 395-416

Orlick (1990). In pursuit of excellence. Human Kinetics, Champaign, IL (1990)

Paivio (1985). Cognitive and motivational functions of imagery in human performance. Canadian Journal of Applied Sport Sciences, 10 (1985), pp. 22S-28S

Reeds (2002). Chronometric comparisons of imagery to action: Visualizing versus physically performing springboard dives. Memory \& Cognition, 30 (8) (2002), pp. 1169-1178

Rushall \& Lippman (1998). The role of imagery in physical performance. International Journal of Sport Psychology, 29 (1998), pp. 57-72

Short et al. (2002). The effect of imagery function and imagery direction on self-efficacy and performance on a golf-putting task. The Sport Psychologist, 16 (2002), pp. 48-67

Short, Monsma, \& Short (2004). Is what you see really what you get? Athletes' perceptions of imagery's functions. The Sport Psychologist, 18 (2004), pp. 341-349

Sparkes (1998). Validity in qualitative inquiry and the problem of criteria: Implications for sport psychology. The Sport Psychologist, 12 (1998), pp. 333-345

Thomas \& Nelson (1996). Research methods in physical activity. Human Kinetics, Champaign, IL (1996) 
Fig. 1. Summary of Munroe et al.'s (2000) conceptual framework for athletes' use of imagery.

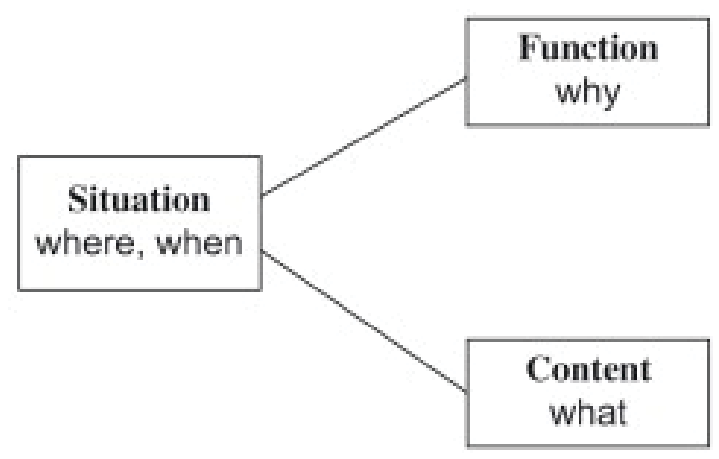

Fig. 2. Alternative working model of athletes' use of imagery.

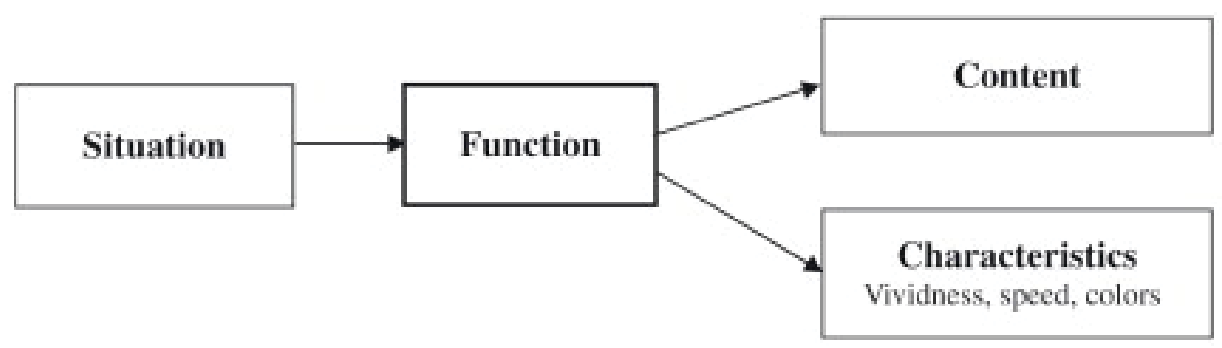

